\title{
APLICAÇÃO DE AGROTÓXICOS EM EUCALIPTO UTILIZANDO PULVERIZADOR PNEUMÁTICO ${ }^{1}$
}

Walter Luis Castro Mewes², Mauri Martins Teixeira ${ }^{3}$, Haroldo Carlos Fernandes ${ }^{3}$, José Cola Zanuncio ${ }^{4}$ e Cleyton Batista de Alvarenga ${ }^{5}$

\begin{abstract}
RESUMO - O trabalho foi desenvolvido visando avaliar os parâmetros da pulverização em culturas arbóreas. A eficiência e a deposição de gotas sobre o alvo são importantes para elevar a rentabilidade na produção de madeira, diminuindo os riscos ambientais, e para a saúde do aplicador, aumentando a eficácia no combate de pragas e doenças. O objetivo deste trabalho foi avaliar os parâmetros operacionais de um pulverizador pneumático para aplicação em florestas adultas de eucalipto, através do estudo da densidade de gotas, da porcentagem de cobertura, do diâmetro da mediana volumétrica, do coeficiente de homogeneidade, da amplitude relativa e da uniformidade de distribuição volumétrica da calda. O pulverizador utilizado mostrou-se eficiente para a aplicação de agrotóxicos em árvores adultas de eucalipto. Observou-se que todos os tratamentos avaliados apresentaram densidade e tamanho de gotas satisfatórias para a pulverização. Quanto aos parâmetros porcentagem de cobertura, deposição e uniformidade de distribuição volumétrica, os melhores resultados ocorreram quando foram utilizados os índices volumétricos de 27 e $20,2 \mathrm{~mL} \mathrm{~m}^{3}$ de dossel. O índice volumétrico de 20,2 $\mathrm{mL}$ $\mathrm{m}^{3}$ de dossel foi o indicado para pulverização.
\end{abstract}

Palavras-chave: Tecnologia de aplicação, Eucaliptus grandis, Volume de copa.

\section{APPLICATION OF PESTICIDE IN EUCALYPTUS SPRAY TYRE}

\begin{abstract}
The study was conducted to evaluate the parameters of the spraying in tree crops. The efficiency and droplet deposition on the target is important to raise the profitability of timber production, reducing environmental risks to health from the applicator, increasing effectiveness in combating pests and diseases. The aim of this study was to evaluate the operational parameters of a pneumatic spray for application to adult eucalypt forests, through the study of droplet density and percentage of coverage, the volume median diameter, the coefficient of homogeneity of relative amplitude and uniform volumetric distribution of the syrup. The sprayer was efficient for the application of pesticides in adult trees of eucalyptus. It was observed that all treatments showed density and droplet size satisfactory for spraying. Regarding the parameters percentage of coverage and uniform volumetric distribution, the best results occurred when we used the volumetric rate of $27 \mathrm{~mL}$ and $20.2 \mathrm{~m}^{3}$ of canopy. As the volume index of $20.2 \mathrm{~mL} \mathrm{~m}$ of canopy suitable for spraying.
\end{abstract}

Keywords: Application technology, Eucalyptus grandis, Crown volume.

\footnotetext{
${ }^{1}$ Recebido em 02.09.2010 aceito para publicação em 05.04.2013.

${ }^{2}$ Universidade Federal de Viçosa, Campus Florestal, UFV, Brasil. E-mail: <walter@ufv.br>.

${ }^{3}$ Departamento de Engenharia Agrícola, Universidade Federal de Viçosa, UFV, Brasil. E-mail:<mauri@ufv.br $>$ e $<$ haroldo@ufv.br $>$.

${ }^{4}$ Centro de Ciências Biológicas e da Saúde, Universidade Federal de Viçosa, UFV, Brasil. E-mail: <zanuncio@ufv.br>.

${ }^{5}$ Universidade Federal de Mato Grosso, UFMT, Brasil. E-mail:<cleytonbatista@yahoo.com.br>.
} 


\section{INTRODUÇÃO}

O Brasil apresentou, nas últimas décadas, invejável evolução na produtividade das florestas de eucaliptos, passando de $15 \mathrm{~m}^{3}$ ha/ano na década de 1970 para, atualmente, uma média nacional de aproximadamente $45 \mathrm{~m}^{3}$ ha/ano (GOMIDE et al., 2010).

As aplicações de agrotóxicos em sistemas florestais têm ganhado destaque com a grande expansão da cultura do eucalipto. Estudos que abordam essa temática devem ser conduzidos visando aumentar a eficiência dessas aplicações em culturas de porte alto e grande densidade foliar, para o controle de insetos desfolhadores como as lagartas em eucalipto.

A pulverização aérea tem sido empregada em diversas áreas de cultivos florestais. Um limitante dessa aplicação é a distância das pistas de reabastecimento. Outro complicador é a declividade acentuada em muitas das regiões cultivadas com florestas de Minas Gerais. Além disso, o custo das aplicações aéreas tem sido, na maioria das vezes, mais elevado que o terrestre, porque as infestações iniciais ocorrem pontualmente e poderiam ser controladas de modo eficiente com a pulverização terrestre. Diante disso, surgem como alternativa bastante viável, dos pontos de vista técnico, econômico e ambiental, as aplicações com pulverizadores pneumáticos.

Os pulverizadores pneumáticos apresentam diâmetro da mediana volumétrica bastante uniforme e geralmente médios, que acompanham facilmente a corrente de ar e se depositam nas folhas. Aplicações terrestres em eucaliptos para controle de lagarta têm a vantagem de serem feitas diretamente no alvo, já que muitos desses insetos têm preferência por folhas mais velhas que se encontram na parte inferior da copa. Desse modo, as aplicações terrestres favorecem o monitoramento intensivo de pragas para controle pontual na lavoura. Já na aplicação aérea as gotas são projetadas na parte superior do dossel e apresentam dificuldades para alcançar o alvo na parte inferior.

O manejo integrado de pragas florestais procura preservar e, ou, aumentar os fatores de mortalidade natural, através do uso de todas as técnicas de controle possíveis, buscando manter a população de pragas abaixo do nível de dano econômico. A adoção do sistema integrado é fundamental nas companhias florestais, pois têm programas de qualidade que buscam melhorar sua eficiência e competitividade. Por exigência do mercado consumidor, priorizam a certificação florestal para atestar a qualidade técnica, ecológica e social das empresas. Com isso, houve a necessidade de se reestruturar os programas de controle florestais, para minimizar os impactos sociais e ambientais negativos de suas atividades. Para conseguir atingir o consumidor, a certificação do manejo florestal requer um sistema que garanta a rastreabilidade da origem de um produto, desde a floresta certificada até o consumidor final (ALVES et al., 2009).

Os pulverizadores são classificados em função da energia utilizada para a fragmentação da calda em gotas, sendo classificados como hidráulicos, hidropneumáticos, pneumáticos, centrífugos e térmicos. No pulverizador pneumático, o fracionamento da calda em pequenas gotas e seu transporte até o alvo ocorrem devido a um fluxo de ar em alta velocidade. Para Fox et al. (2008), à medida que as gotas se afastam do pulverizador, há diminuição da velocidade do fluxo de ar, e este vai se tornando desuniforme e espalhando-se durante sua trajetória. As gotas pequenas tendem a seguir a corrente aerodinâmica do fluxo de ar, depositando-se no alvo pelo movimento turbulento, enquanto as gotas grandes se depositam principalmente pelo impacto.

As gotas pequenas podem, em alguns casos, ser afetadas pela corrente de ar responsável pelo seu transporte e pelas condições meteorológicas (velocidade do vento, temperatura e umidade relativa do ar). No entanto, a penetração de gotas grandes no interior das copas pode ser reduzida por causa das folhas e pelo atrito com o ar. Para a distribuição da calda na planta, vários fatores estão envolvidos, como tamanho da árvore, volume da copa, deriva, tamanho das gotas, volume de calda, velocidade e sentido de deslocamento do pulverizador e do vento (SCUDELER et al., 2004).

Desse modo, no processo de pulverização em florestas adultas o depósito de gotas na copa das árvores pode ser afetado pela arquitetura da planta e pela densidade foliar. Até então, não se tem conhecimento de um pulverizador terrestre capaz de lançar as gotas em copas acima de $20 \mathrm{~m}$ com boa deposição e uniformidade. Como consequência, obtêm-se baixa eficácia de controle, aumento no volume da calda, contaminação ambiental e aumento do custo da produção (PAI et al., 2008). 
Para alcançar distribuição uniforme de calda no dossel de plantas arbóreas, diminuir a agressão ao meio ambiente e aumentar a eficiência das aplicações, devem-se torná-las pontuais quanto ao local e momento correto de execução. Marti et al. (2006), avaliando a otimização da aplicação de agrotóxicos em plantas arbóreas, concluíram que a distribuição uniforme é difícil de ser alcançada, objetivando sempre aumentar a penetração de gotas e o desenvolvimento dos pulverizadores pneumáticos. Afirmam ainda que dificilmente se conseguem deposições uniformes em plantas arbóreas, e o uso de pulverizadores com assistência de ar é alternativa bastante eficaz na busca por pulverizações mais eficientes. Nesse caso, o volume de calda deve ser diretamente relacionado às características da planta, como volume e densidade foliar, e não em função de um volume estimado de calda.

Os fabricantes têm lançado no mercado inúmeros modelos de pontas de pulverização, com o intuito de elevar a qualidade das aplicações (KNEWITZ et al., 2002). Entretanto, para a pulverização pneumática, o cenário é diferente. Atualmente, encontram-se diversos modelos desses pulverizadores em uso, mas com poucas informações básicas sobre o espectro de gotas que esse equipamento produz em determinadas condições meteorológicas.

As pulverizações em culturas de porte alto apresentam, na maioria das vezes, muita desuniformidade em relação à distribuição da calda. Os inseticidas protetores necessitam de contato com o alvo para serem eficazes no controle. Os insetos se instalam em locais de difícil deposição, reduzindo o intervalo entre as pulverizações em função do efeito negativo da má distribuição da calda. Nesse caso, além de baixa eficácia de controle, há o risco de ocorrer seleção de biótipos resistentes de insetos ou fungos (KONNO et al., 2001).

Assim, o objetivo deste trabalho foi avaliar os parâmetros técnicos da pulverização pneumática em florestas adultas de eucalipto, através do estudo do índice volumétrico, do espectro e da população de gotas da pulverização, visando à melhor eficiência e à segurança socioambiental das aplicações.

\section{MATERIAL E MÉTODOS}

O trabalho foi desenvolvido no Laboratório de Mecanização da Universidade Federal de Viçosa, Viçosa, MG, localizado na latitude de $20^{\circ} 43^{\prime} \mathrm{S}$, longitude $42^{\circ}$
51 'O e altitude de $640 \mathrm{~m}$. No experimento, foi utilizado um pulverizador pneumático, marca Jacto, modelo Jatão 600 , acionado por um trator Valtra 800.

Inicialmente, foi desenvolvida uma estrutura de prolongação com altura de $14 \mathrm{~m}$. Para complementar a altura até $21 \mathrm{~m}$, foi construída uma estrutura metálica de tubos galvanizados de forma telescópica, de modo a permitir a colocação de etiquetas hidrossensíveis nas alturas desejadas.

Utilizou-se um tubo de 12,70 mm de diâmetro com $6 \mathrm{~m}$ de comprimento. Em sua extremidade, soldou-se um tubo de mesmo diâmetro e com 3,5 m de comprimento, formando um ângulo de $90^{\circ}$, onde foram fixadas quatro roldanas equidistantes. Foi usado um segundo tubo de $3 \mathrm{~m}$ de comprimento e 19,05 mm de diâmetro, com parafusos soldados na sua parede, de forma que o primeiro tubo pudesse ser introduzido nesse segundo e fixado pelos parafusos. Foi utilizado, ainda, um terceiro tubo de 1,5 m de comprimento e 25,5 mm de diâmetro, também com parafusos fixados em sua parede, para elevar a estrutura até $21 \mathrm{~m}$ de altura.

Essa estrutura foi montada em um viaduto para facilitar a amostragem das pulverizações. Com a ajuda das roldanas foram colocadas cordas, e nestas foram afixadas as etiquetas hidrossensíveis em sete alturas diferentes $(3,6,9,12,15,18$ e $21 \mathrm{~m})$, considerando-se a estrada sob o viaduto como referência.

A eficiência da distribuição da calda pulverizada foi avaliada utilizando-se etiquetas hidrossensíveis, marca Syngenta, de 38 x $26 \mathrm{~mm}$. As etiquetas foram distribuídas a cada $3 \mathrm{~m}$ de altura, em todos os cabos. Assim, mediram-se a densidade de gotas e a porcentagem de cobertura na etiqueta. Para a determinação do tamanho das gotas, considerou-se o fator de espalhamento (equação 1), proposto por Chaim et al. (1999).

$\mathrm{F}=0,74057+0,0001010399 \mathrm{D}+0,2024884 \ln (\mathrm{D})$

em que F é o fator de espalhamento e D é o diâmetro limite de cada classe de tamanho da mancha $(\mu \mathrm{m})$.

As aplicações foram realizadas utilizando-se três volumes de calda e levando em consideração o volume da copa das árvores, previamente estimados, e os índices volumétricos já consagrados para pulverização em culturas arbóreas (VIRGINIA, 1989). A avaliação dos parâmetros da pulverização foi realizada considerando-se a porcentagem de cobertura, densidade de gotas, diâmetro da mediana volumétrica e amplitude relativa.

Revista Árvore, Viçosa-MG, v.37, n.2, p.347-353, 2013 
Em função da posição de deslocamento do trator em relação às etiquetas e com o objetivo de que o jato atingisse as etiquetas superiores, usou-se uma inclinação de $15^{\circ}$ do bocal atomizador do pulverizador em relação à vertical. $\mathrm{O}$ volume de aplicação foi definido com base no método do volume do dossel das árvores (SUTTON; UNRATH, 1984, 1988), considerando as médias do diâmetro, da altura de copa e do espaçamento entre fileiras de plantio (equação 2).

$$
T R V=\frac{H L}{D} 10000
$$

em que:

TRV = volume do dossel das plantas por área $\left(\mathrm{m}^{3} \mathrm{ha}^{-1}\right)$;

$\mathrm{H}=$ altura do dossel (m);

$\mathrm{L}=$ largura do dossel (m); e

$\mathrm{D}=$ distância entre fileiras de plantio $(\mathrm{m})$.

Após a pulverização, as etiquetas amostradoras foram acondicionadas individualmente de acordo com a altura (3, 6, 9, 12, 15, 18 e $21 \mathrm{~m})$ e levadas ao laboratório para análises. As etiquetas foram digitalizadas utilizando-se Scaner HP, modelo Scanjet 3770, na resolução de 1.200 dpi e analisadas com o auxílio do software IMAGE TOOL, versão 3.0, conforme Ruas (2007) e Magno Júnior (2008).

Para determinação da uniformidade de deposição de calda, utilizaram-se etiquetas plásticas com $20 \mathrm{x}$ $30 \mathrm{~mm}$, colocadas nas mesmas alturas e posições das etiquetas hidrossensíveis. Após a aplicação da calda, as etiquetas plásticas foram recolhidas e acondicionadas individualmente em sacos plásticos, de acordo com sua altura e levadas ao laboratório para análise em espectrofotômetro, determinando-se, assim, a quantidade de produto que atingiu cada etiqueta.

A curva de calibração do espectrofotômetro foi obtida a partir da solução-padrão $\left(2 \mathrm{~g} \mathrm{~L}^{-1}\right)$ em várias concentrações conhecidas do traçador azul brilhante (FD \& C Blue $n^{\circ} 1$ ). Foram feitas leituras de absorbância no espectrofotômetro e determinada a curva de calibração, utilizando-se uma amostra da calda aplicada no campo (Figura 1). A solução foi analisada em espectrofotômetro modelo SP-1105, determinando-se a absorbância com o uso de uma radiação eletromagnética de 630 nm, conforme descrito por Palladini (2005) e Scudeler et al. (2004).

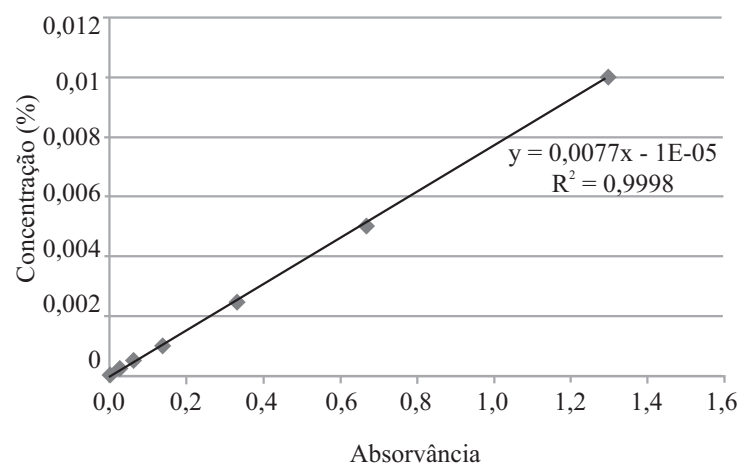

Figura 1 - Curva de calibração do espectrofotômetro. Figure 1-Calibration curve of the spectrophotometer.

As etiquetas plásticas recolhidas foram lavadas em 50 mL de água destilada e, dessa solução, retirou-se uma amostra para determinação da concentração do traçador através da leitura da absorbância. A partir desse valor, determinou-se o volume de calda na etiqueta plástica. Durante o experimento, foram monitoradas as condições de velocidade do vento, temperatura e umidade relativa do ar.

O experimento foi conduzido em parcelas subdivididas, tendo nas parcelas três volumes de aplicação e, nas subparcelas, sete alturas, em delineamento de blocos casualizados com três repetições, totalizando 63 unidades experimentais.

\section{RESULTADOS}

Durante o experimento, a temperatura do ar variou entre 25 e $27^{\circ} \mathrm{C}$, a velocidade do vento de 0 a $1,0 \mathrm{~m} \mathrm{~s}^{-1}$ a $2 \mathrm{~m}$ do solo, enquanto a umidade relativa permaneceu acima de $75 \%$. Os volumes de calda obtidos para as velocidades de deslocamento de 2,4; 3,3; e 5,3 $\mathrm{km} \mathrm{h}^{-1}$, foram de 2.754, 2.066 e $1035 \mathrm{~L} \mathrm{ha}^{-1}$, respectivamente. As velocidades de deslocamento foram obtidas nas três primeiras marchas. A vazão da calda foi de $33,2 \mathrm{~L} \mathrm{~min}^{-1}$. Os índices volumétricos foram de 27,0,20,2 e 12,8 $\mathrm{mL} \mathrm{m}^{3}$, obtidos nas respectivas velocidades.

A vazão de ar obtida foi de $11.300 \mathrm{~m}^{3} \mathrm{~h}^{-1}$, utilizando-se a rotação-padrão de $540 \mathrm{rpm}$ na tomada de potência (TDP) do trator. Os dados foram analisados pelo teste F e de Tukey, adotando-se o nível de significância de $5 \%$ de probabilidade.

De acordo com a análise de variância, observou-se que houve interação significativa entre a altura da etiqueta e o volume de pulverização do parâmetro densidade 
de gotas. Pode-se observar que os melhores resultados foram obtidos nas alturas de 12,15 e $18 \mathrm{~m}$, nos três índices volumétricos avaliados (Tabela 1 ).

O volume de calda afetou o diâmetro da mediana volumétrica. O tamanho das gotas foi sempre maior que $100 \mu \mathrm{m}$ (Tabela 2).

A amplitude relativa ou SPAN é uma referência ao estudo da variação dos diâmetros das gotas pulverizadas. A interação volume versus altura foi significativa para amplitude relativa (Tabela 3 ).

Tabela 1 - População de gotas $\left(\right.$ gotas $\mathrm{cm}^{-2}$ ) obtidas nas diferentes alturas de aplicação e índices volumétricos $\left(\mathrm{mL} \mathrm{m}^{-3}\right)$.

Table 1 - Population drops (drops $\mathrm{cm}^{-2}$ ) obtained at different times of application and volumetric rates $\left(\mathrm{mL} \mathrm{m}^{-3}\right)$.

\begin{tabular}{cccccccc}
\hline \multirow{2}{*}{ IVmL m $^{-3}$} & \multicolumn{7}{c}{ Altura (m) } \\
\cline { 2 - 8 } & 3 & 6 & 9 & 12 & 15 & 18 & 21 \\
\hline 27,0 & $17,0 \mathrm{c}$ & $37,7 \mathrm{c}$ & $62,7 \mathrm{c}$ & $225,0 \mathrm{~b}$ & $245,3 \mathrm{ab}$ & $320,3 \mathrm{a}$ & $244,0 \mathrm{ab}$ \\
20,2 & $22,8 \mathrm{~d}$ & $191,0 \mathrm{c}$ & $308,3 \mathrm{~b}$ & $416,3 \mathrm{a}$ & $289,7 \mathrm{~b}$ & $286,0 \mathrm{~b}$ & $106 \mathrm{~cd}$ \\
12,8 & $29,0 \mathrm{ab}$ & $10,7 \mathrm{~b}$ & $19,7 \mathrm{~b}$ & $93,3 \mathrm{ab}$ & $116,7 \mathrm{a}$ & $45,7 \mathrm{ab}$ & $14,3 \mathrm{~b}$ \\
\hline
\end{tabular}

Médias seguidas pela mesma letra minúscula nas linhas não diferem, a $5 \%$ de probabilidade, pelo teste de Tukey.

Tabela 2 - Diâmetro da mediana volumétrica $(\mu \mathrm{m})$ em relação a altura das etiquetas e índice volumétrico (IV).

Table 2 - Volume median diameter ( $\mu \mathrm{m})$ over the height of the labels and volume index (IV)

\begin{tabular}{cccccccc}
\hline \multirow{2}{*}{ IVmL m $^{-3}$} & \multicolumn{7}{c}{ Altura (m) } \\
\cline { 3 - 8 } & 3 & 6 & 9 & 12 & 15 & 18 & 21 \\
\hline 27,0 & $276,7 \mathrm{ab}$ & $268,7 \mathrm{ab}$ & $321,2 \mathrm{a}$ & $316,2 \mathrm{a}$ & $278,2 \mathrm{ab}$ & $227,0 \mathrm{~b}$ & $251,4 \mathrm{~b}$ \\
20,2 & $233,9 \mathrm{a}$ & $200,4 \mathrm{a}$ & $223,4 \mathrm{a}$ & $191,2 \mathrm{a}$ & $243,0 \mathrm{a}$ & $208,9 \mathrm{a}$ & $201,1 \mathrm{a}$ \\
12,8 & $134,2 \mathrm{~b}$ & $226,1 \mathrm{a}$ & $187,5 \mathrm{ab}$ & $186,2 \mathrm{ab}$ & $208,0 \mathrm{a}$ & $226,8 \mathrm{a}$ & $228,1 \mathrm{a}$ \\
\hline
\end{tabular}

Médias seguidas pela mesma letra minúscula nas linhas não diferem, a $5 \%$ de probabilidade, pelo teste de Tukey.

Tabela 3 - Amplitude relativa em relação à altura das etiquetas e índice volumétrico (IV).

Table 3 - Relative amplitude in relation to height of the labels and volume index $(I V)$.

\begin{tabular}{cccccccc}
\hline \multirow{2}{*}{$\mathrm{IV} \mathrm{mL} \mathrm{m}^{-3}$} & \multicolumn{7}{c}{ Altura (m) } \\
\cline { 2 - 8 } & 3 & 6 & 9 & 12 & 15 & 18 & 21 \\
\hline 27,0 & $0,8 \mathrm{ab}$ & $0,7 \mathrm{~b}$ & $1,1 \mathrm{ab}$ & $1,1 \mathrm{ab}$ & $1,2^{\mathrm{a}}$ & $1,1 \mathrm{ab}$ & $1,1 \mathrm{ab}$ \\
20,2 & $0,6 \mathrm{c}$ & $0,9 \mathrm{bc}$ & $1,0 \mathrm{abc}$ & $1,2 \mathrm{ab}$ & $1,3 \mathrm{ab}$ & $1,4 \mathrm{a}$ & $1,1 \mathrm{ab}$ \\
12,8 & $1,2 \mathrm{a}$ & $0,7 \mathrm{~b}$ & $0,8 \mathrm{ab}$ & $1,0 \mathrm{ab}$ & $0,9 \mathrm{ab}$ & $0,8 \mathrm{ab}$ & $0,7 \mathrm{~b}$ \\
\hline
\end{tabular}

Médias seguidas pela mesma letra minúscula nas linhas não diferem, a $5 \%$ de probabilidade, pelo teste de Tukey.
Os depósitos de calda nas folhas foram influenciados pela interação altura e volume. O volume de calda acumulado na folha foi expresso em $\mu \mathrm{L} \mathrm{cm}^{2}$ (Tabela 4).

\section{DISCUSSÃO}

As maiores densidades de gotas foram obtidas na faixa de 12 a $18 \mathrm{~m}$ de altura para todos os volumes. Entretanto, aos $21 \mathrm{~m}$ o número de gotas foi maior que o indicado para controle de doenças causadas por fungos e insetos, como demonstrado por Barthelemy et al. (1990). Para inseticida, necessita-se de 20 a 30 gotas $\mathrm{cm}^{2}$ e para fungicidas, de 50 a 70 gotas por $\mathrm{cm}^{2}$. Ainda de acordo com o recomendado, apenas nas alturas de 6,9 e $21 \mathrm{~m}$, utilizando o menor índice volumétrico, a população de gotas é insuficiente para controle de insetos e fungos.

Esse equipamento apresenta-se como excelente alternativa para aplicação de inseticida na forma de bacilo (Bacillus thurigiensis) a ser ingerida pelas lagartas, pois apresentou percentual de cobertura na faixa de 10 a $20 \%$ e população de gotas satisfatória, nos índices volumétricos de 27 e $20,2 \mathrm{~mL} \mathrm{~m}^{3}$ de copa. A população de gotas é diretamente proporcional ao percentual de cobertura, que é fator preponderante na aplicação dos bacilos e também um parâmetro utilizado para avaliação da qualidade da pulverização, uma vez que é muito difícil conseguir número adequado de gotas nas condições de plantas altas e copas densas.

Pulverizações com gotas abaixo de $100 \mu \mathrm{m}$ são muito suscetíveis à deriva. Já gotas muito acima de $300 \mu \mathrm{m}$ podem favorecer o escorrimento da calda e a consequente perda para o solo. Em culturas de copas densas, como eucalipto, o tamanho de gotas ganha ainda mais importância, visto que as correntes de ar produzidas pelos pulverizadores pneumáticos são as

Tabela 4 - Calda depositada $\left(\mu \mathrm{L} \mathrm{cm}^{-2}\right)$ em relação a altura das etiquetas e índice volumétrico (IV).

Table 4 - Spray deposited $\left(\mathrm{mL} \mathrm{cm}^{-2}\right)$ over the height of the labels and volume index (IV).

\begin{tabular}{cccccccc}
\hline \multirow{2}{*}{ IV mL m $^{-3}$} & \multicolumn{8}{c}{ Altura (m) } \\
\cline { 2 - 8 } & 3 & 6 & 9 & 12 & 15 & 18 & 21 \\
\hline 27,0 & $0,1 \mathrm{~d}$ & $0,1 \mathrm{~d}$ & $0,6 \mathrm{~cd}$ & $1,9 \mathrm{c}$ & $3,6 \mathrm{~b}$ & $4,8 \mathrm{ab}$ & $6,2 \mathrm{a}$ \\
20,2 & $1,0 \mathrm{bc}$ & $0,5 \mathrm{c}$ & $1,3 \mathrm{bc}$ & $3,2^{\mathrm{a}}$ & $2,1 \mathrm{ab}$ & $2,4 \mathrm{ab}$ & $0,2 \mathrm{c}$ \\
12,8 & $0,1 \mathrm{a}$ & $0,2 \mathrm{a}$ & $0,2 \mathrm{a}$ & $0,06^{\mathrm{a}}$ & $0,2 \mathrm{a}$ & $0,1 \mathrm{a}$ & $0,2 \mathrm{a}$ \\
\hline
\end{tabular}

Médias seguidas pela mesma letra minúscula nas linhas não diferem, a $5 \%$ de probabilidade, pelo teste de Tukey.

Revista Árvore, Viçosa-MG, v.37, n.2, p.347-353, 2013 
responsáveis por conduzir as gotas até o interior da copa. Desse modo, o emprego de gotas grandes reduz a eficiência das pulverizações.

O diâmetro da mediana volumétrica é um indicador pontual da qualidade da pulverização. Portanto, não deve ser usado isoladamente pelo responsável técnico como determinante para selecionar pontas ou avaliar uma aplicação de agrotóxicos.

Com os índices volumétricos de 27,0 e 20,8 mL m³ de dossel, constatou-se redução nos valores de amplitude relativa nas menores alturas. Em função da configuração de projeto do canhão direcionado para cima, as gotas pequenas e mais uniformes ficavam em menores alturas, e as gotas médias e grandes eram transportadas pela corrente de ar até a copa.

Os melhores valores de SPAN tendem a zero, indicando não haver grande variação entre as classes de diâmetros das gotas. Porém, como o pulverizador pneumático utilizado tinha sua saída direcionada para aplicação em grandes alturas, o foco era atingir as etiquetas de 15,18 e $21 \mathrm{~m}$ de altura. As etiquetas mais baixas provavelmente receberam, em sua maioria, gotas menores provenientes de deriva. A amplitude relativa nos dá uma ideia melhor da aplicação e deve ser analisada juntamente com o tamanho de gotas para caracterizar a pulverização.

O volume de calda depositado nos alvos é o parâmetro mais científico para determinação da qualidade da aplicação, porque, utilizando a espectrofometria, determina-se exatamente quanto de produto ficou retido no alvo. Os maiores valores depositados foram observados nas alturas intermediárias, para o maior índice volumétrico, semelhante aos resultados encontrados por Ramos et al. (2007) em citros.

Vale ressaltar que ainda devem ser conduzidos estudos que analisem se há e quais seriam as interações dos produtos com os corantes utilizados e que indiquem uma faixa de segurança de volume por unidade de área foliar em que é necessário controlar esta ou aquela praga neste ou naquele estádio de desenvolvimento. Vários autores já têm apontado alguns parâmetros a serem abordados na escolha do melhor corante, como Palladini et al. (2005), que estudaram a relação de diversos corantes com a calda.

Revista Árvore, Viçosa-MG, v.37, n.2, p.347-353, 2013

\section{CONCLUSÕES}

$\mathrm{O}$ experimento mostrou que o pulverizador pneumático pode ser utilizado de forma eficiente para aplicação de fitossanitários em árvores adultas de eucalipto.

A densidade de 244 e 106 gotas $\mathrm{cm}^{-2}$ a $21 \mathrm{~m}$ de altura foi considerada suficiente para o controle de insetos no eucalipto.

O maior diâmetro da mediana volumétrica $(321,2 \mu \mathrm{m})$ e o menor (134,2 $\mu \mathrm{m})$ não comprometeram a qualidade das pulverizações

O índice volumétrico de $20,2 \mathrm{~mL} \mathrm{~m}^{3}$ de dossel apresentou melhores parâmetros médios para pulverização.

\section{REFERÊNCIAS}

ALVES, R. R. et al. Certificação florestal e o mercado moveleiro nacional. Revista Árvore, v.33, n.3, p.583-589, 2009.

BARThELEMY, P. et al. Choisir les outils de pulverisation. Paris: Institut Technique des Céréales et des Fourrages, 1990. 160p.

CHAIM, A.; MAIA, A. H. N.; PESSOA, M. C. P. Y. Estimativa da deposição de agrotóxicos por análise de gotas. Pesquisa Agropecuária Brasileira, v.34, n.6, p.963-969, 1999.

FOX, R. D. et al. A history of air-blast sprayer development and future prospects.

Transactions of the ASABE, v. 51, n.2, p.405-410, 2008.

GOMIDE, J. L.; FANTUZZI NETO, H.; REGAZZI, A. J. Análise de critérios de qualidade da madeira de eucalipto para produção de celulose Kraft. Revista Árvore, v.34, n.2, p.339-344, 2010.

KNEWITZ, H.; WEISSER, P.; KOCH, H. Driftreducing spray application in orchards and biological efficacy of pesticides. Aspects of Applied Biology, v.66, p.231-236, 2002. (Número Especial).

KONNO, R. H.; FRANCO, C. R.; OMOTO, C. Suscetibilidade de populações de Brevipalpus phoenicis (Geijskes, 1939) (Acari: Tenuipalpidae) a acaricidas organoestânicos em citros.

Scientia Agrícola, v.58, n.4, p.703-709, 2001. 
MAGNO JÚNIOR, R. G. Desenvolvimento de sistema eletrônico para pulverização e atração de gotas em plantas cítricas. 2008. 69f. Dissertação (Mestrado em Engenharia Agrícola) - Universidade Federal de Viçosa, Viçosa, MG, 2008.

MARTI, S. P. et al. Optimizing pesticide spray application in tree crops. St. Joseph: ASABE, 2006. (Paper, 1128).

PAI, N.; SALYANI, M.; SWEEB, R. D. Adjusting airblast sprayer airflow based on tree foliage density. St. Joseph: ASAE, 2008. (Paper, 3653).

PALLADINI, L. A.; RAETANO, C. G.; VELINI, E. D. Choice of tracers for the evaluation of spray deposits. Scientia Agrícola, v.62, n.5, p.440-445, 2005.

RAMOS, H. H. et al. Características da pulverização e citros em função do volume de calda aplicado com turbopulverizador.

Engenharia Agrícola, v.27, n.1, p.56-65, 2007.

RUAS, R. A. A. Tecnologia de aplicação de glyphosate para certificação de produtos agrícolas. 2007. 107f. Tese (Doutorado em Engenharia Agrícola) Universidade Federal de Viçosa, Viçosa, MG, 2007.
SCUDELER, F. et al. Cobertura da pulverização e maturação de frutos do cafeeiro com ethephon em diferentes condições operacionais. Bragantia, v.63, n.1, p.129-139, 2004.

SUTTON, T. B.; UNRATH, C. R. Evaluation of the tree-row volume concept with density adjustments in relation to spray deposition in apple orchards. Plant Disease, v.68, n.6, p.480-484, 1984.

SUTTON, T. B.; UNRATH, C. R. Evaluation of the tree-row-volume model for full season pesticide application on apples. Plant Disease, v.72, n.7, p.629-632, 1988.

TSAY, J. et al. Evaluation of a pneumatic-shielded spraying system by CFD simulation. Transactions of the ASAE, v.45, n.1, p.47-54, 2002.

VIRGINIA AND WEST WIRGINIA COOPERATIVE EXTENSION SERVICES 1989-1990 Spray Bulletin for Commercial Tree Fruit Growers. Morgantown: West Virginia University, 1989. 117p. (Publ. 456-419). 